VISUAL EXPERIMENT

\title{
Histopathological analysis of 2,4,5-trinitrobenzene sulphonic acid (TNBS)-induced mouse colitis
}

\author{
Kang-Ho Choi, Hong Hyeonju and Irfan A. Rather \\ Department of Applied Microbiology and Biotechnology, Yeungnam University, Gyeongsan 712-749, South \\ Korea.
}

Correspondence to Irfan A. Rather at rather@ynu.ac.kr

First Published: 12 June, 2016

DOI: 10.3329/bjp.v11i3.28155

\section{ABSTRACT}

Ulcerative colitis is one of the chronic diseases that results in inflammation of colon in humans. In this Visual experiment we demonstrate the histopathology of TNBS induced colitis model of mouse. The experiment involves following steps: a) Selection of mouse model, b) Induction of colitis, c) Paraffin sectioning of colon tissue, d) Hematoxylin and Eosin staining, and d) Microscopic observation.

\section{INTRODUCTION}

Ulcerative colitis is a type of inflammatory bowel disease (IBD) that has affected millions of people throughout the world. Till date, there is no known cause for IBD. Therefore, it is difficult to find out positive treatments. 2,4,5-trinitrobenzene sulphonic acid (TNBS)- induced ulcerative colitis is one of the best The animal model of ulcerative colitis resembles to that of human. Diagnosis of colitis is one of the key features and histopathological examination plays a major role in the diagnosis and management of ulcerative colitis. In general, ulcerative colitis is characterized by inflammation particularly colonic mucosa. The mucosal inflammation involves the rectum and different lengths of the proximal colon. The distal colon is severely swollen than the proximal colon. Ulcerative colitis can be proctitis, left-side colitis, sub-total colitis, and pancolitis (Satsangi et al., 2006). The proctitis is limited to the lining of the rectum, called the rectal mucosa, left-side colitis starts at the rectum and extends up the left colon, sub-total colitis extended up to the splenic flexure, whereas pancolitis is the extensive colitis of entire colon. Therefore, histological analysis of ulcerative colitis tissue is one of the basic diagnostic requirements. The key features for diagnosis are the architecture of crypt, crypt density, transmucosal lamina propia cell increase, diffuse of basal plasmacytosis. In addition, increased intensity of the alterations towards the distal colon could be seen in most cases. Overall, ulcerative colitis is characterized by inflammation and architectural distortion from proximal towards the distal colon and examination of multiple tissue samples is needed for proper assessment.

\section{MATERIALS AND EQUIPMENTS}

- TNBS

- Ethanol

- Susceptible strain of 5- to 6-week-old mice (males) of known weight

- Inhalable anesthetic (e.g. ethyl ether)

- Surgical lubricant (if available)

- 3.5-French 38-cm, polyurethane catheter

- 1-mL disposable syringe

- Diethyl ether

- Cotton pads 
- Glass chamber for anesthesia

- Gloves

- Eye care glasses

- $\mathrm{NaCl}$

- Distilled water

- 1x PBS

- Ethanol $(30 \%, 50 \%, 60 \%, 70 \%)$, diluted with double distilled water

- Glass vials with screw on lids

- Orbital rocker

- Hematoxylin

- Eosin

- Permount

- Concentrated hydrochloric acid

- Confocal microscope

\section{PREPARATION OF REAGENTS}

- Preparation of saline at physiological $\mathrm{pH}$ : Add $0.45 \mathrm{~g}$ of $\mathrm{NaCl}$ in $50 \mathrm{~mL}$ distilled water.

- Preparation of 50\% ethanol in saline: Mix absolute alcohol (99.9\%) and saline in 1:1 ratio.

- Preparation of TNBS: Earlier studies have demonstrated that a dose of TNBS at an amount ranging in between $0.4 \mathrm{mg}$ to $4.0 \mathrm{mg}$ per $\mathrm{kg}$ weight of mouse prepared in 30 to $50 \%$ ethanol, was successful in inducing colitis. The appropriate amount (i.e., $0.4 \mathrm{mg}$ to $4.0 \mathrm{mg}$ in 30 to $50 \%$ ethanol) should be instilled in a total volume of $100 \mu \mathrm{L}$ to $150 \mu \mathrm{L}$ per mouse.

\section{VIDEO CLIPS}

Part 1: $\quad 13 \min 14 \mathrm{sec}$

Part 2: $\quad 3 \min 13 \mathrm{sec}$

\section{METHOD}

Selection of mouse model: ICR mice are the best mice models of ulcerative colitis. It is recommended to use at least 6 to 7 weeks old mice. The mice were occupied from Samtaco Bio Korea. The mice were allowed to eat standard chow pellet diet and maintained on a 12 hours light/dark cycle with relative humidity of $30-70 \%$. The procedure was approved by the animal care committee at Yeungnam University.

Induction of ulcerative colitis: A $100 \mu \mathrm{L}$ per mouse of TNBS was administered through rectal rout for the induction of colitis. A total of six mice were used per group.

Mice observation and confirmation of colitis: After induction of TNBS-induced colitis, mice were monitored for bleeding, diarrhea, watery stool, urine color and/or any other injury.

Protocol for induction of colitis: Please follow our previously published VE for details about induction of TNBS-induced colitis (Rather et al. 2015).

\section{Histological process}

The selected regions of individual colon samples are crossly trimmed based on the lumen. After proper washing, and paraffin embedding, 3-4 $\mu \mathrm{m}$ sections are prepared. The selected sections are strained with hematoxylin and eosin (H\&E) for light microscopy examination. However, in addition to H\&E, other stains such as Masson's trichrome for collagen fibers, or toluidine blue for mast cells could be also used.

\section{Paraffin embedding protocol}

Day 1:

1. Wash tissues using $1 \times$ PBS three times for $30 \mathrm{~min}$

2. Dehydrate tissue using following washes

2.1. $30 \%$ ethanol for 1 hour at room temperature

2.2. $50 \%$ ethanol for 1 hour at room temperature

2.3. $60 \%$ ethanol for 1 hour at room temperature 
2.4. $70 \%$ ethanol for 1 hour at room temperature

2.5. $70 \%$ ethanol overnight at room temperature

Note: If time allows, do longer 1x PBS washes. Use at least 200 proof ethanol. Do not leave tissues exposed to air.

Day 2-3

Use tissues from the overnight wash in $70 \%$ ethanol made on Day 1 and follow the steps as under:

1. Wash with $85 \%$ ethanol for 1 hour at room temperature

2. $95 \%$ ethanol for 1 hour at room temperature

3. $100 \%$ ethanol for $30 \mathrm{~min}$ at room temperature

4. $100 \%$ ethanol for 1 hour at room temperature

5. 1:1 xylene: ethanol for 1 hour at room temperature

6. $100 \%$ xylene for $30 \mathrm{~min}$ at room temperature

7. $100 \%$ xylene for 1 hour at room temperature

8. 1:1 xylene: paraffin for 1 hour at $60-65^{\circ} \mathrm{C}$ in bead trays (small flask) on paraffin oven

9. $100 \%$ Paraffin 1 hour $60-65^{\circ} \mathrm{C}$ in bead trays on paraffin oven

10. $100 \%$ Paraffin 3 hours $60-65^{\circ} \mathrm{C}$ in bead trays on paraffin oven

11. $100 \%$ Paraffin 3 hours $60-65^{\circ} \mathrm{C}$ in bead trays on paraffin oven

12. $100 \%$ Paraffin 3 hours $60-65^{\circ} \mathrm{C}$ in bead trays on paraffin oven

13. $100 \%$ Paraffin overnights $60-65^{\circ} \mathrm{C}$ in bead trays on paraffin oven

Day 4

1. Fill clear paraffin mold with paraffin. Rest on heated surface

2. Take tissues out from paraffin container (flask) and quickly put tissues in the paraffin molds on center. Arrange tissue in correct orientation in warm paraffin

3. Add a little bit of warm paraffin to top of clear mold. Swirl around, then quickly press embedding ring onto the clear mold. Fill the rest of the mold to the top of the embedding ring

4. Move mold to unheated surface and let paraffin harden slightly while maintaining correct position of tissues

5. Let harden on bench top. Samples will keep in the mold at RT overnight

Day 5- Sectioning

1. Heat water in water tray. Turn light on while using the water tray. Let water heat for at least 1 hour before needed

2. Clean blade and around cutting surface with a kimwipe. Let dry completely

3. Cut off excess wax from embedded sample. Cut off excess wax from embedding ring. Can also trim around sample if desired

4. Lock microtome blade in place and make sure blade guards are closed. Lock microtome handle and clamp embedded sample onto microtome

5. Adjust sample position so it sits straight and even right above the blade. Lock into place

6. Unlock and quickly turn handle until sample starts cutting a little. Try to capture the first full section using wooden probe to encourage future sections to stick to each other and create a ribbon of paraffin sections. Use 5-10 micron (using 5 micron for best staining result) thickness for sections

7. Gently place cut sections into warm water tray using tweezers and metal probe

8. Use metal probe to gently maneuver the sections onto a glass slide

9. Let slide dry upright in drying rack until most of the moisture is gone

10. Transfer slides to adjust platform to finish drying. Leave on platform overnight on air 
11. Store in plastic slide carrier/slide box until ready to stain

Hematoxylin and eosin (H\&E) staining

(Modified From Baylor College of Medicine)

- Place slides containing paraffin sections in a slide holder (glass or metal)

-Deparaffinize and rehydrate sections:

1. $3 \times 3^{\prime}$ Xylene (blot excess xylene before going into ethanol)

2. $3 \times 3^{-} 100 \%$ ethanol

3. $1 \times 3^{\prime} 95 \%$ ethanol

4. $1 \times 5^{\prime} \mathrm{PBS}$

- While sections are in PBS, skim surface of hematoxalin with a Kimwipe to remove oxidized particles. Blot excess water from slide holder before going into hematoxalin

- Hematoxalin staining:

1. $1 \times 3^{\prime}$ Hematoxalin

2. Rinse PBS

3. Dip 4-5x (fast) acid ethanol (to destain)

4. Dip 4-5x (fast) ammonia water $(0.3 \%)$

- Eosin staining and dehydration:

1. $1 \times 3-5^{\prime}$ Eosin (up to $5 \mathrm{~min}$ for an older batch of eosin)

2. $3 \times 3^{\prime} 95 \%$ ethanol

3. $3 \times 3^{\prime} 100 \%$ ethanol (blot excess ethanol before going into xylene)

4. $3 \times 3^{\prime}$ Xylene

5. Coverslip slides using permount (xylene based)

6. Place a drop of permount on the slide using a glass rod, taking care to leave no bubbles

7. Angle the coverslip and let fall gently onto the slide. Allow the permount to spread beneath the coverslip, covering all the tissue

8. Dry overnight in the hood

\section{DISCUSSION}

The severity of ulcerative colitis can be scored based on the histopathology and histomorphometry, and have been provided valuable criteria (Stucchi et al., 2000; Peran et al., 2005; Park et al., 2012). The positive results are considered as an evidence of inflammation. In addition, the efficacy of anti-inflammatory drugs can be also monitored based on the scoring of UC.

Table 1. Criteria for Assessment of Microscopic Colonic Damage (Histological Scoring Systems)

Mucosal epithelium

1. Ulceration: none (0); mild - surface (1); moderate (2); extensive-full thickness (3)

Crypts

2. Mitotic activity: lower third (0); mild mid-third (1); moderate mid-third (2); upper third (3)

3. Mucus depletion: none (0); mild (1); moderate (2); severe (3)

Lamina propria 
4. Inflammatory cell infiltrate: none (0); mild (1); moderate (2); severe (3)

5. Vascularity: none (0); mild (1); moderate (2); severe (3)

6. Collagen depositions: none (0); mild (1); moderate (2); severe (3)

Submucosal

7. Inflammatory cell infiltrate: none (0); mild (1); moderate (2); severe (3)

8. Collagen depositions: none (0); mild (1); moderate (2); severe (3)

9. Vascularity: none (0); mild (1); moderate (2); severe (3)

10. Edema: none (0); mild (1); moderate (2); severe (3)

Maximum score: 30. Modified from Stucchi et al, (2000) and Peran et al, (2005)

\section{REFERENCES}

Park SY, Ku SK, Lee ES, Kim JA. 1,3-Diphenylpropenone ameliorates TNBS-induced rat colitis through suppression of NF-kB activation and IL-8 induction. Chem Biol Interact. 2012; 196: 39-49.

Peran L, Camuesco D, Comalada M, Nieto A, Concha A, Diaz-Ropero MP, Olivares M, Xaus J, Zarzuelo A, Galvez J. Preventative effects of a probiotic, Lactobacillus salivarius ssp. salivarius, in the TNBS model of rat colitis. World J Gastroenterol. 2005; 11: 5185-92.

Rather IA, Bajpai VR, Gyeong-Jun N. Mouse model of ulcerative colitis using trinitrobenzene sulfonic acid. Bangladesh J Pharmacol. 2015; 10: 860-63.

Satsangi J, Silverberg MS, Vermeire S, et al. The Montreal classification of inflammatory bowel disease: Controversies, consensus, and implication. Gut 2006; 55: 749-53.

Stucchi AF, Shofer S, Leeman S, Materne O, Beer E, McClung J, Shebani K, Moore F, O'Brien M, Becker JM. NK-1 antagonist reduces colonic inflammation and oxidative stress in dextran sulfate-induced colitis in rats. Am J Physiol Gastrointest Liver Physiol. 2000; 279: 1298-306.

\section{PRECAUTION}

TNBS is very hazardous and exposure to any part of body should be presented using gloves, lab coat, and eye care glasses

Please avoid colon tissue to air dry during ethanol washing 


\section{Your feedback about this paper}

1. Number of times you have read this paper 0

2. Number of times you have seen the video clip 0

3. Quality of paper Click

4. Your comments

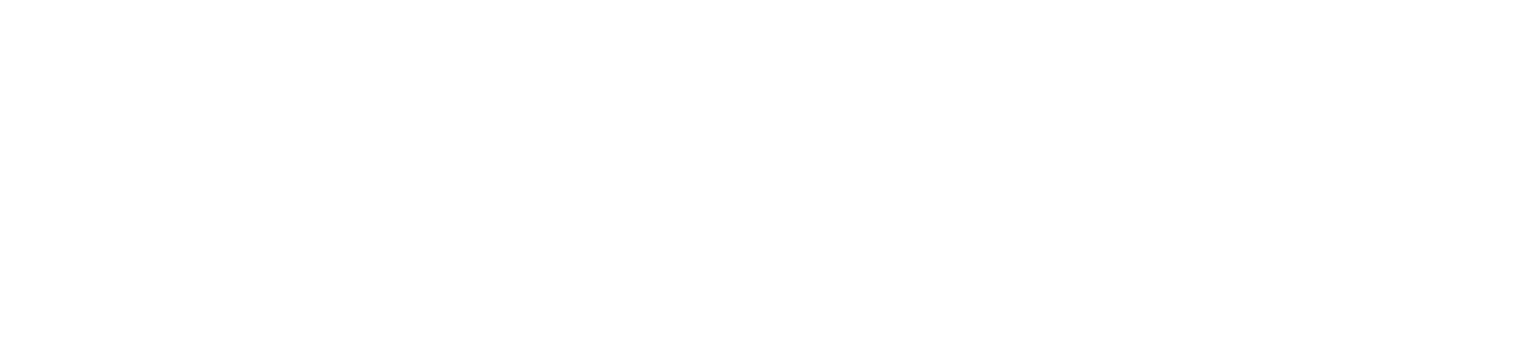

